\title{
Study the Correlation Coefficient and Path Coefficient for the yield and yield Component of Bitter Gourd (Momordica charantia L.)
}

\author{
Deepak Maurya $^{1}$, V.B. Singh ${ }^{1}$, G.C. Yadav ${ }^{1}$, VeerendraKumar ${ }^{1}$, \\ Shivam Dubey ${ }^{2}$ and Ashok Kumar Pandey ${ }^{3}$
}

\author{
${ }^{1}$ Department of Vegetable Science, ${ }^{2}$ Department of GPB, Narendra Deva University of \\ Agriculture and Technology, Kumarganj, Faizabad- 224 229, (U.P.), India \\ ${ }^{3}$ Department of Horticulture, DRMLU, Faizabad-224 001, U. P. (India)
}

*Corresponding author

\section{Keywords \\ Path-coefficient, Correlation analysis, Bitter gourd genotypes, etc.}

\section{Article Info}

Accepted:

10 January 2019

Available Online:

10 February 2019

\section{A B S T R A C T}

The present investigation entitled "Study the correlation coefficient and path coefficient for the yield and yield component of bitter gourd (Momordica charantia L.) was carried out at Main Experiment Station, Department of Vegetable Science, Narendra Deva University of Agriculture \& Technology, Kumarganj, Faizabad (U.P.) during summer2017, to evaluate the available genotypes, to estimate the correlation coefficient and to work out the path coefficient analysis for yield and its component traits of 30genotypes, with three replications in randomized block design. Estimates of correlation and path coefficients can define the mutual relationship between plant characters and determine component characters on which selection can be based for improvement in yield. It might be easier to increase yield by increasing the smallest yield components on an otherwise good cultivar. Thirty genotypes of bitter gourd were evaluated for yield contributing characters to observe their associations and direct and indirect effect on fruit yield. In most cases the genotypic correlation coefficient was higher than the respective phenotypic correlation coefficients indicating the suppressive effect of environment modified phenotypic expression of these characters by reducing phenotypic correlation values. The higher magnitude of coefficient of variation at phenotypic as well as genotypic levels were observed for phenotypic in no. of fruit per plant followed by fruit yield per plant $(\mathrm{kg})$, vinelength $(\mathrm{m})$, fruit length $(\mathrm{cm})$, node no. to anthesis of first staminate flower, average fruit weight (g), node no. to anthesis of first pistillate flower, no. of nodes per vine and lower value in days of first fruit harvest followed by days to anthesis of first pistillate flower, days to anthesis of first staminate flower, fruit diameter. The phenotypic correlation coefficients between different characters were generally similar in magnitude and nature to the corresponding genotypic correlation coefficient. The significant and positive correlation with yield per plant was observed at phenotypic level with average fruit weight and no. of fruits per plant. The analysis of path coefficient indicating appreciable amount of direct positively effect of no. of fruits per plant and fruit yield per plant followed by vine length on fruit yield per plant. 


\section{Introduction}

Bitter gourd (Momordica charantia L.) offers a high degreeof variation for developing cultivars with desirable qualitative traits, and tolerance to biotic and abiotic yield limiting factors. The immature fruits are highly nutritious (Gopalan et al., 1982) and a rich source of phosphorus $(55 \mathrm{mg} / 100 \mathrm{~g})$, iron (1.8 $\mathrm{mg} / 100 \mathrm{~g}$ ), calcium (20 mg/100 g), vitamin C $(88 \mathrm{mg} / 100 \mathrm{~g})$ and vitamin A (219 IU/100 g). Whole bitter gourd plants are used in medicinal preparations (Morton 1967).

Correlation, in general, measures the extent and direction (positive or negative) of a relationship occurring between 2 or more characteristics (Gomez and Gomez 1984, Rohman et al., 2003). Simple correlation describes the overall relationship between 2 or more characteristics, whereas estimates of genetic and phenotypic correlations describe the extent of genetic and phenotypic factors in establishing a relationship between two plant traits. The estimate of genetic correlation (rg) refers to the association between 2 plant characters due to the genetic constitution of the plant, phenotypic correlation (rp) refers to the correlation between 2 plant characters due to their physical appearance at a morphological, anatomical, or biochemical level (Affifi, 1984, Kang 1998, Zhang et al., 2005). Path analysis was adopted in plant breeding experiments by Dewey and $\mathrm{Lu}$ (1959), and has been used extensively in agronomic and environmental studies (Garcia del Moral et al., 2003; Zhang et al., 2005). It is a standardized partial regression analysis measuring, the direct influence of 1 variable upon another and permits separation of correlation into direct and indirect effects. Path analysis is useful for determining the contribution of component variables to a character (Rafi and Nath, 2004; Zhang et al., 2005; Carlos et al., 2005). The undertaken to determine the nature of association, direct and indirect relationship between yield and yield contributing characters, and the relative contribution of each character towards fruit yield in bitter gourd through character association and path coefficient analysis.

\section{Materials and Methods}

Thirty diverse bitter gourd genotypes including check (Pusa Do Mausami) were used. The experiment was arranged in randomized complete block design with 3 replications during (spring-summer) of 2017 at the Main Experiment Station (Vegetable Research Farm), Narendra Deva University of Agriculture and Technology, Kumarganj, Faizabad, India (26.47 ${ }^{\circ}$ North latitude and $82.12^{\circ}$ East longitudes at an altitude of $113 \mathrm{~m}$ above the mean sea level). Plot size $3 \times 2 \mathrm{~m}$ with a row to row spacing of $2 \mathrm{~m}$ and plant to plant spacing of $0.50 \mathrm{~m}$. Observations 5 randomly selected plants from each genotype in each replication were made for node number to anthesis of first staminate flower, node number to anthesis of first pistillate flower, days to anthesis of first staminate flower, days to anthesis of first pistillate flower, days to first fruit harvest, vine length, number of nodes per vine, fruit length, fruit diameter, average fruit weight, number of fruits per plant and fruit yield per plant. Genotypic and phenotypic correlations were calculated per Al-Jibouri et al., (1958) using an ANOVA and covariance matrix in which total variability was split into replications, genotypes, and errors. Genotypic and phenotypic correlation coefficients were used to determine direct and indirect contribution toward yield per plot. The direct and indirect paths were obtained according to the method of Dewey and Lu (1959).

\section{Results and Discussion}

The correlation coefficients among characters were determined at the phenotypic and 
genotypic levels. Genotypic correlation coefficients were higher in magnitude than phenotypic correlation coefficients. This indicates a strong inherent genotypic relationship between characters studied, through the phenotypic expression was impeded by environmental influence.

Node number to anthesis of first pistillate flower showed significant and positive association with the node number to anthesis of first staminate flower $(\mathrm{rp}=0.571)$, days to anthesis of first staminate flower showed significant and positive association with node number to anthesis of first staminate flower $(\mathrm{rp}=0.395)$ and node number to anthesis of first pistillate flower ( $\mathrm{rp}=0.172$ ); days to anthesis of first pistillate flower with node number to anthesis of first staminate flower $(\mathrm{rp}=0.541)$, days to anthesis of first staminate flower $(\mathrm{rp}=0.535)$ and node number to anthesis of first pistillate flower $(r p=0.415)$, days to first fruit harvest showed with node number to anthesis of first staminate flower $(\mathrm{rp}=0.357)$, node number to anthesis of first pistillate flower $(\mathrm{rp}=0.159)$, days to anthesis of first staminate flower $(\mathrm{rp}=0.613)$ and days to anthesis of first pistillate flower ( $\mathrm{rp}=$ $0.673)$.

Vine length (m) showed high significant and positive association with node number to anthesis of first staminate flower $(\mathrm{rp}=0.217)$, no. of nodes per vine $(\mathrm{rp}=0.468)$. fruit diameter $(\mathrm{cm})$ showed high significant and positive association with days to first fruit harvest ( $\mathrm{rp}=0.273)$; $\mathrm{C}$

No. of fruit per plant showed high significant and positive association with days to anthesis of first staminate flower $(\mathrm{rp}=0.226)$. Fruit yield per plant $(\mathrm{kg})$ showed significant and positive association with fruit weight $(\mathrm{rp}=0.676)$; no. of fruits per plant $(\mathrm{rp}=0.313)$ in Table 1.
While, positive and non-significant correlation was observed in nodes per vine with days to anthesis of first staminate flower $(\mathrm{rp}=0.009)$, no. of fruit per plant positive and non-significant correlation with days to anthesis of first staminate flower ( $r p=0.004)$, fruit diameter anthesis of first staminate flower, fruit length ( $\mathrm{rp}=0.122)$ and fruit diameter positive and non-significant correlation with fruit length $(\mathrm{rp}=0.174)$ and days to first fruit harvest shows positive and non-significant correlation with node no. of first pistillate flower $(\mathrm{rp}=0.159)$.

Whereas, negative and non-significant correlation was observed in average fruit weight with fruit diameter $(\mathrm{rp}=-0.0147)$ and node no. of first pistillate flower $(\mathrm{rp}=$ 0.0287), no. of fruits per plant showed negative and non-significant correlation with fruit length $(\mathrm{rp}=-0.019)$ and node no. of first pistillate flower $(\mathrm{rp}=-0.020)$, fruit length showed negative and non-significant correlation with vine length $(\mathrm{rp}=-0.035)$ and days to anthesis of first staminate flower ( $\mathrm{rp}=$ -0.086), vine length showed negative and non-significant correlation with days to first fruit harvest $(\mathrm{rp}=-0.106)$ and days to anthesis of first pistillate flower ( $\mathrm{rp}=-0.164)$, no. of node per vine showed negative and nonsignificant correlation with days to first fruit harvest $(\mathrm{rp}=-0.059)$ and days to anthesis of first pistillate flower $(\mathrm{rp}=-0.156)$.

In contrast, path coefficient analysis permits a critical examination of specific direct and indirect effects of characters and measures the relative importance of each of them in determining the ultimate goal yield. Path coefficients analysis was estimated on phenotypic as well as genotypic levels (Table 2) to resolve the direct and indirect effects of different characters on fruit yield per plant. 
Table.1 Estimates of the phenotypic correlation coefficient between twelve characters in bitter gourd genotype

\begin{tabular}{|c|c|c|c|c|c|c|c|c|c|c|c|c|c|}
\hline $\begin{array}{l}\text { S. } \\
\text { No }\end{array}$ & Character & $\begin{array}{c}\text { Node no. of } \\
\text { 1st staminate } \\
\text { flower } \\
\text { appearance }\end{array}$ & $\begin{array}{c}\text { Node no. of } \\
\text { 1st pistillate } \\
\text { flower } \\
\text { appearance }\end{array}$ & $\begin{array}{c}\text { Days to } \\
\text { anthesis of 1st } \\
\text { staminate } \\
\text { flower } \\
\text { appearance }\end{array}$ & $\begin{array}{c}\text { Days to } \\
\text { anthesis of } \\
1 \text { st } \\
\text { pistillate } \\
\text { flower }\end{array}$ & $\begin{array}{c}\text { Days to 1st } \\
\text { fruit } \\
\text { harvest }\end{array}$ & $\begin{array}{l}\text { Nodes per } \\
\text { vine. }\end{array}$ & $\begin{array}{c}\text { Vine } \\
\text { length }(\mathrm{m})\end{array}$ & $\begin{array}{l}\text { Fruit } \\
\text { length } \\
(\mathbf{c m})\end{array}$ & $\begin{array}{c}\text { Fruit } \\
\text { diameter } \\
\text { (cm) }\end{array}$ & $\begin{array}{c}\text { No. of } \\
\text { fruits per } \\
\text { plant }\end{array}$ & $\begin{array}{c}\text { Fruit } \\
\text { weight } \\
\text { (gm) }\end{array}$ & $\begin{array}{c}\text { Marktable } \\
\text { fruit } \\
\text { yield/plant } \\
\text { (kg) }\end{array}$ \\
\hline 1. & $\begin{array}{l}\text { Node no. of 1st } \\
\text { staminate flower } \\
\text { appearance }\end{array}$ & 1.0000 & $0.5717 * *$ & $0.3957 * *$ & $0.5411 * *$ & $0.3571 * *$ & -0.0748 & $0.2176^{*}$ & $-0.2599 *$ & -0.0439 & 0.0191 & $\begin{array}{l}- \\
0.3876^{*} \\
\quad *\end{array}$ & $\begin{array}{c}- \\
0.3616^{*} \\
*\end{array}$ \\
\hline 2. & $\begin{array}{l}\text { Node no. of 1st } \\
\text { pistillate flower } \\
\text { appearance }\end{array}$ & & 1.0000 & $0.1728 * *$ & $0.4153 * *$ & 0.1593 & $\begin{array}{l}- \\
0.3652 * \\
*\end{array}$ & -0.1469 & $-0.2250 *$ & -0.0801 & -0.2040 & -0.0287 & $-0.2499 *$ \\
\hline 3. & $\begin{array}{l}\text { Days to anthesis of } \\
\text { 1st staminate flower } \\
\text { appearance }\end{array}$ & & & 1.0000 & $0.5351 * *$ & $0.6139 * *$ & 0.0098 & -0.1517 & -0.0866 & $0.2693^{*}$ & $0.2265^{*}$ & $\begin{array}{c}- \\
0.3938 * \\
*\end{array}$ & -0.1514 \\
\hline 4. & $\begin{array}{l}\text { Days to anthesis of } \\
\text { 1st pistillate flower } \\
\text { appearance }\end{array}$ & & & & 1.0000 & $0.6731 * *$ & -0.1560 & -0.1648 & $-0.2372 *$ & 0.1999 & 0.0411 & $-0.3143^{*}$ & -0.2583 \\
\hline 5. & $\begin{array}{l}\text { Days to 1st fruit } \\
\text { harvest }\end{array}$ & & & & & 1.0000 & -0.0597 & -0.1064 & $-0.2593 *$ & $\begin{array}{c}0.2735^{*} \\
*\end{array}$ & 0.1380 & $\begin{array}{c}- \\
0.3874 * \\
*\end{array}$ & $-0.2819^{*}$ \\
\hline 6. & Nodes per vine. & & & & & & 1.0000 & $\begin{array}{c}0.4683^{*} \\
*\end{array}$ & 0.0486 & 0.0713 & $\begin{array}{c}0.0730 * \\
*\end{array}$ & $-0.2431^{*}$ & -0.2268 \\
\hline 7. & Vine length (m) & & & & & & & 1.0000 & -0.0351 & 0.0045 & 0.0240 & -0.1165 & -0.1440 \\
\hline 8. & Fruit length $(\mathrm{cm})$ & & & & & & & & 1.0000 & 0.1747 & -0.0190 & 0.1269 & 0.0934 \\
\hline 9. & Fruit diameter $(\mathrm{cm})$ & & & & & & & & & 1.0000 & -0.0294 & -0.0147 & 0.0486 \\
\hline 10 & Fruits per plant & & & & & & & & & & 1.0000 & $\begin{array}{l}- \\
0.3430 * \\
*\end{array}$ & $0.3137^{*}$ \\
\hline 11 & Fruit weight (gm) & & & & & & & & & & & 1.0000 & $\begin{array}{r}0.6761^{*} \\
*\end{array}$ \\
\hline
\end{tabular}

* - Significant at 5 percent probability level

** - Significant at 1 percent probability level 
Table.2 Estimates of the genotypic correlation coefficient between twelve characters in bitter gourd genotypes

\begin{tabular}{|c|c|c|c|c|c|c|c|c|c|c|c|c|c|}
\hline S.no. & Character & $\begin{array}{c}\text { Node no. of } \\
\text { 1st } \\
\text { staminate } \\
\text { flower } \\
\text { appearance }\end{array}$ & $\begin{array}{c}\text { Node no. of } \\
\text { 1st pistillate } \\
\text { flower } \\
\text { appearance }\end{array}$ & $\begin{array}{c}\text { Days to } \\
\text { anthesis of } \\
\text { 1st } \\
\text { staminate } \\
\text { flower } \\
\text { appearance }\end{array}$ & $\begin{array}{c}\text { Days to } \\
\text { anthesis } \\
\text { of 1st } \\
\text { pistillate } \\
\text { flower }\end{array}$ & $\begin{array}{c}\text { Days } \\
\text { to 1st } \\
\text { fruit } \\
\text { harvest }\end{array}$ & $\begin{array}{c}\text { No. } \\
\text { of } \\
\text { Nodes } \\
\text { per } \\
\text { vine }\end{array}$ & $\begin{array}{l}\text { Vine } \\
\text { length } \\
(\mathbf{m})\end{array}$ & $\begin{array}{c}\text { Fruit } \\
\text { length } \\
(\mathrm{cm})\end{array}$ & $\begin{array}{c}\text { Fruit } \\
\text { diameter } \\
(\mathrm{cm})\end{array}$ & $\begin{array}{c}\text { Fruits } \\
\text { per } \\
\text { plant }\end{array}$ & $\begin{array}{c}\text { Fruit } \\
\text { weight } \\
(\mathrm{gm})\end{array}$ & $\begin{array}{c}\text { Market } \\
\text { able } \\
\text { fruit } \\
\text { yield/pla } \\
\text { nt }(\mathbf{k g})\end{array}$ \\
\hline 1. & $\begin{array}{l}\text { Node no. of 1st } \\
\text { staminate flower } \\
\text { appearance }\end{array}$ & 1.0000 & 0.7138 & 0.5974 & 0.6725 & 0.5764 & -0.1220 & 0.2423 & -0.2608 & -0.0731 & 0.1061 & -0.4522 & -0.4458 \\
\hline 2. & $\begin{array}{l}\text { Node no. of } 1 \text { st pistillate } \\
\text { flower appearance }\end{array}$ & & 1.0000 & 0.3200 & 0.6543 & 0.3860 & -0.4675 & -0.1924 & -0.3157 & -0.1193 & -0.2031 & -0.0610 & -0.2966 \\
\hline 3. & $\begin{array}{l}\text { Days to anthesis of 1st } \\
\text { staminate flower } \\
\text { appearance }\end{array}$ & & & 1.0000 & 0.8989 & 0.8654 & 0.0134 & -0.1544 & -0.1975 & 0.4652 & 0.3574 & -0.5182 & -0.2086 \\
\hline 4. & $\begin{array}{l}\text { Days to anthesis of 1st } \\
\text { pistillate flower } \\
\text { appearance }\end{array}$ & & & & 1.0000 & 0.9849 & -0.2290 & -0.2198 & -0.2430 & 0.3977 & 0.0045 & -0.3812 & -0.3217 \\
\hline 5. & Days to 1 st fruit harvest & & & & & 1.0000 & -0.0836 & -0.1921 & -0.2970 & 0.6118 & 0.0841 & -0.4774 & -0.2847 \\
\hline 6. & No.of nodes per vine. & & & & & & 1.0000 & 0.5204 & 0.0951 & 0.0533 & 0.1129 & -0.2864 & -0.2559 \\
\hline 7. & Vine length (m) & & & & & & & 1.0000 & -0.0555 & 0.0136 & 0.0325 & -0.1047 & -0.1290 \\
\hline 8. & Fruit length $(\mathrm{cm})$ & & & & & & & & 1.0000 & 0.2230 & -0.0181 & 0.1116 & 0.1144 \\
\hline 9. & Fruit diameter $(\mathrm{cm})$ & & & & & & & & & 1.0000 & -0.0448 & -0.0307 & 0.0484 \\
\hline 10. & No. of fruits per plant & & & & & & & & & & 1.0000 & -0.3900 & 0.3974 \\
\hline 11. & $\begin{array}{l}\text { Average fruit weight } \\
\text { (gm) }\end{array}$ & & & & & & & & & & & 1.0000 & 0.7094 \\
\hline
\end{tabular}


Table.3 Direct and indirect effects of twelve characters of fruit yield/ plant (kg) at phenotypic level in bitter gourd

\begin{tabular}{|c|c|c|c|c|c|c|c|c|c|c|c|c|c|}
\hline $\begin{array}{l}\text { S. } \\
\text { No. }\end{array}$ & Character & $\begin{array}{c}\text { Node no. of } \\
\text { 1st } \\
\text { staminate } \\
\text { flower } \\
\text { appearance }\end{array}$ & $\begin{array}{l}\text { Node no. of } \\
\text { 1st pistillate } \\
\text { flower } \\
\text { appearance }\end{array}$ & $\begin{array}{c}\text { Days to } \\
\text { anthesis of } \\
\text { 1st } \\
\text { staminate } \\
\text { flower } \\
\text { appearance }\end{array}$ & $\begin{array}{c}\text { Days to } \\
\text { anthesis of } \\
1 \text { st } \\
\text { pistillate } \\
\text { flower }\end{array}$ & \begin{tabular}{|} 
Days to 1st \\
fruit \\
harvest
\end{tabular} & 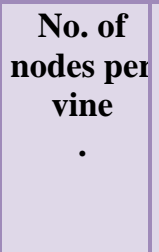 & $\begin{array}{c}\text { Vine } \\
\text { length } \\
\text { (m) }\end{array}$ & $\begin{array}{c}\text { Fruit } \\
\text { length } \\
\text { (cm) }\end{array}$ & $\begin{array}{c}\text { Fruit } \\
\text { diameter } \\
(\mathbf{c m})\end{array}$ & $\begin{array}{c}\text { No. of } \\
\text { fruits per } \\
\text { plant }\end{array}$ & $\begin{array}{c}\text { Average } \\
\text { fruit } \\
\text { weight } \\
\text { (gm) }\end{array}$ & $\begin{array}{l}\text { Fruit } \\
\text { yield } \\
\text { per } \\
\text { plant } \\
\text { (kg) }\end{array}$ \\
\hline 1. & $\begin{array}{l}\text { Node no. of 1st staminate flower } \\
\text { appearance }\end{array}$ & 0.0629 & 0.0360 & 0.0249 & 0.0341 & 0.0225 & -0.0047 & 0.0137 & -0.0164 & -0.0028 & 0.0012 & -0.0244 & -0.3616 \\
\hline 2. & $\begin{array}{l}\text { Node no. of } 1 \text { st pistillate flower } \\
\text { appearance }\end{array}$ & -0.1200 & -0.2099 & -0.0363 & -0.0872 & -0.0334 & 0.0767 & 0.0308 & 0.0472 & 0.0168 & 0.0428 & 0.0060 & -0.2499 \\
\hline 3. & $\begin{array}{l}\text { Days to anthesis of } 1 \text { st staminate } \\
\text { flower appearance }\end{array}$ & 0.0469 & 0.0205 & 0.1185 & 0.0634 & 0.0728 & 0.0012 & -0.0180 & -0.0103 & 0.0319 & 0.0268 & -0.0467 & -0.1514 \\
\hline 4. & $\begin{array}{l}\text { Days to anthesis of } 1 \text { st pistillate } \\
\text { flower }\end{array}$ & 0.0138 & 0.0106 & 0.0137 & 0.0256 & 0.0172 & -0.0040 & -0.0042 & -0.0061 & 0.0051 & 0.0011 & -0.0080 & -0.2583 \\
\hline 5. & Days to 1 st fruit harvest & -0.0586 & -0.0261 & -0.1008 & -0.1105 & -0.1641 & 0.0098 & 0.0175 & 0.0426 & -0.0449 & -0.0227 & 0.0636 & -0.2819 \\
\hline 6. & No. of nodes per vine. & 0.0091 & 0.0444 & -0.0012 & 0.0190 & 0.0073 & -0.1217 & -0.0570 & -0.0059 & -0.0087 & -0.0089 & 0.0296 & -0.2268 \\
\hline 7. & Vine length (m) & -0.0095 & 0.0064 & 0.0066 & 0.0072 & 0.0046 & -0.0205 & -0.0437 & 0.0015 & -0.0002 & -0.0010 & 0.0051 & -0.1440 \\
\hline 8. & Fruit length $(\mathrm{cm})$ & 0.0191 & 0.0166 & 0.0064 & 0.0175 & 0.0191 & -0.0036 & 0.0026 & -0.0736 & -0.0129 & 0.0014 & -0.0093 & 0.0934 \\
\hline 9. & Fruit diameter $(\mathrm{cm})$ & -0.0041 & -0.0075 & 0.0252 & 0.0187 & 0.0256 & 0.0067 & 0.0004 & 0.0163 & 0.0935 & -0.0027 & -0.0014 & 0.0486 \\
\hline 10. & No.of fruits per plant & 0.0109 & -0.1162 & 0.1290 & 0.0234 & 0.0786 & 0.0416 & 0.0137 & -0.0108 & -0.0167 & 0.5697 & -0.1954 & 0.3137 \\
\hline 11. & Average fruit weight (gm) & -0.3322 & -0.0246 & -0.3375 & -0.2694 & -0.3320 & -0.2083 & -0.0998 & 0.1088 & -0.0126 & -0.2940 & 0.8570 & 0.6761 \\
\hline
\end{tabular}

Residual effect $=0.3987$ 
Table.4 Direct and indirect effects of twelve characters of fruit yield/ plant (kg) at a genotypic level in bitter gourd

\begin{tabular}{|c|c|c|c|c|c|c|c|c|c|c|c|c|c|}
\hline No & Character & $\begin{array}{l}\text { Node no. of } \\
\text { 1st staminate } \\
\text { flower } \\
\text { appearance }\end{array}$ & $\begin{array}{c}\text { Node no. of } \\
\text { 1st } \\
\text { pistillate } \\
\text { flower } \\
\text { appearance }\end{array}$ & $\begin{array}{c}\text { Days to } \\
\text { anthesis of } \\
\text { 1st } \\
\text { staminate } \\
\text { flower } \\
\text { appearance }\end{array}$ & $\begin{array}{l}\text { Days to } \\
\text { anthesis } \\
\text { of } 1 s t \\
\text { pistillate } \\
\text { flower }\end{array}$ & $\begin{array}{c}\text { Days } \\
\text { to 1st } \\
\text { fruit } \\
\text { harvest }\end{array}$ & $\begin{array}{c}\text { No. of } \\
\text { nodes } \\
\text { per } \\
\text { vine. }\end{array}$ & $\begin{array}{c}\text { Vine } \\
\text { length } \\
\text { (m) }\end{array}$ & $\begin{array}{l}\text { Fruit } \\
\text { length } \\
\text { (cm) }\end{array}$ & $\begin{array}{c}\text { Fruit } \\
\text { diameter } \\
(\mathbf{c m})\end{array}$ & $\begin{array}{c}\text { No. of } \\
\text { fruits } \\
\text { per } \\
\text { plant }\end{array}$ & $\begin{array}{l}\text { Average } \\
\text { fruit } \\
\text { weight } \\
\text { (gm) }\end{array}$ & $\begin{array}{l}\text { Fruit } \\
\text { yield } \\
\text { per } \\
\text { plant } \\
\text { (kg) }\end{array}$ \\
\hline 1. & $\begin{array}{c}\text { Node no. of 1st staminate flower } \\
\text { appearance }\end{array}$ & -0.1466 & -0.1046 & -0.0876 & -0.0986 & -0.0845 & 0.0179 & -0.0355 & 0.0382 & 0.0107 & -0.0155 & 0.0663 & -0.4458 \\
\hline 2. & $\begin{array}{c}\text { Node no. of } 1 \text { st pistillate flower } \\
\text { appearance }\end{array}$ & -0.2132 & -0.2987 & -0.0956 & -0.1954 & -0.1153 & 0.1396 & 0.0575 & 0.0943 & 0.0356 & 0.0607 & 0.0182 & -0.2966 \\
\hline 3. & $\begin{array}{l}\text { Days to anthesis of } 1 \text { st staminate } \\
\text { flower appearance }\end{array}$ & -0.3274 & -0.1754 & -0.5480 & -0.4926 & -0.4743 & -0.0074 & 0.0846 & 0.1082 & -0.2550 & -0.1959 & 0.2840 & -0.2086 \\
\hline 4. & $\begin{array}{c}\text { Days to anthesis of } 1 \text { st pistillate } \\
\text { flower }\end{array}$ & 0.3817 & 0.3714 & 0.5102 & 0.5676 & 0.5590 & -0.1300 & -0.1248 & -0.1379 & 0.2257 & 0.0026 & -0.2164 & -0.3217 \\
\hline 5. & Days to 1 st fruit harvest & 0.2194 & 0.1470 & 0.3295 & 0.3750 & 0.3807 & -0.0318 & -0.0731 & -0.1131 & 0.2329 & 0.0320 & -0.1818 & -0.2847 \\
\hline 6. & Nodes per vine. & 0.0112 & 0.0428 & -0.0012 & 0.0209 & 0.0076 & -0.0915 & -0.0476 & -0.0087 & -0.0049 & -0.0103 & 0.0262 & -0.2559 \\
\hline 7. & Vine length (m) & 0.0237 & -0.0188 & -0.0151 & -0.0215 & -0.0188 & 0.0509 & 0.0978 & -0.0054 & 0.0013 & 0.0032 & -0.0102 & -0.1290 \\
\hline 8. & Fruit length $(\mathrm{cm})$ & -0.0173 & -0.0210 & -0.0131 & -0.0161 & -0.0197 & 0.0063 & -0.0037 & 0.0664 & 0.0148 & -0.0012 & 0.0074 & 0.1144 \\
\hline 9. & Fruit diameter $(\mathrm{cm})$ & 0.0101 & 0.0165 & -0.0642 & -0.0549 & -0.0845 & -0.0074 & -0.0019 & -0.0308 & -0.1381 & 0.0062 & 0.0042 & 0.0484 \\
\hline 10. & Fruits per plant & 0.0992 & -0.1900 & 0.3343 & 0.0042 & 0.0787 & 0.1056 & 0.0304 & -0.0169 & -0.0419 & 0.9354 & -0.3648 & 0.3974 \\
\hline 11. & Average fruit weight (gm) & -0.4867 & -0.0657 & -0.5577 & -0.4103 & -0.5138 & -0.3083 & -0.1127 & 0.1201 & -0.0330 & -0.4198 & 1.0763 & 0.7094 \\
\hline
\end{tabular}


Maximum positive direct effect on fruit yield per plant recorded by average fruit weight (0.857) followed by no. of fruits per plant (0.569), days to anthesis of first staminate flower (0.118), fruit diameter (0.093) (Table 3 and 4).

Negative direct effect on fruit yield per plant showed by vine length (-0.043), fruit length ($0.0736)$, no. of nodes per vine $(-0.121)$, days to first fruit harvest $(-0.164)$, node number of first staminate flower (-0.209), however, the direct effect of the rest of characters were too low to consider of any consequence.

Highest positive indirect effect showed by average fruit weight (0.108) via. Fruit length. Followed by no. of fruits per plant had an indirect positive effect on fruit yield per plant via days to anthesis of first staminate flower (0.129) followed by days to first fruit harvest (0.078).

The negative indirect effect average fruit weight on fruit yield per plant, via. Days to anthesis of first staminate flower $(-0.337)$, node no. of first staminate flower $(-0.332)$, days to first fruit harvest (-0.332) and no. of fruits per plant $(-0.294)$. while average fruit weight on fruit yield per plant, via. no. of nodes per vine (-0.208) had a negative indirect effect on fruit yield per plant. Rest of the estimates of indirect effect was too low.

\section{References}

Afifi AA. Computer-Aided Multivariate Analysis; Lifetime Learning Publications: Belmont, Calif, 1984.

Al-Jibouri HA, Millar PA, Robinson HF. Genotypic and environmental variances and co-variances in an upland cotton cross of interspecific origin. Agronomy Journal. 1958; 50:633-636.

Bhave SG, Bendale VW, Pethe UB, Berde
SA, Mehta JL. Correlation and path analysis in segregating generations of bitter gourd. Journal of Soils and Crops. 2003; 13(1):33-40.

Carlos AFS, Senalik D, Simon PW. Path analysis suggests phytoene accumulation is the key step limiting the carotenoid pathway in white carrot roots. Genetics and Molecular Biology. 2005; 28(2):287-293.

Dewey OR, Lu KH. Correlation and path coefficient analysis of components of crested wheatgrass seed production. Journal of Agronomy. 1959; 51:515518.

Dey SS, Behera TK, Pal A, Munshi AD. Correlation and path coefficient analysis in bitter gourd (Momordica charantia L.). Vegetable Science. 2005; 32(2):173-176.

Garcia del Moral LF, Rharraabti Y, Villages D, Royo C. Evaluation of grain yield and its components in durum wheat under Mediterranean conditions: $A n$ ontogenic approach. Agronomy Journal. 2003; 95: 266-274.

Gomez KA, Gomez AA. Statistical Procedures for Agricultural Research. John Willey and Sons: New York, 1984.

Gopalan C, Ramashastri BV, Balasubramanian SC. Nutritive value of Indian foods. I. C. M. R., Hyderabad. 1982, 328.

Grubben GJH. Tropical vegetable and their genetic resources, IBPGR, Rome. 1977, 51-52.

Gupta N, Bhardwaj ML, Singh SP, Sood S. Correlation and path analysis of yield and yield components in some genetic stocks of bitter gourd (Momordica charantia L.). SABRAO Journal of Breeding and Genetics. 2015; 47(4):475-481.

Hazra P, Som MG. Vegetable Science. 2nd Revised Edition. Kalyani Publishers, 
New Delhi. 2015, 327.

Islam MR, Hossain MS, Bhuiyan MSR, Husna A, Syed MA. Genetic variability and path-coefficient analysis of bitter gourd (Momordica charantiaL.). International Journal of Sustainable Agriculture. 2009; 1(3):53-57.

Kang MS. Using genotype-by-environment interaction for crop cultivar development. Advances in Agronomy. 1998; 62:199-251.

Morton JF. The balsam pear-an edible medicinal and toxic plant. Economic Botany. 1967; 212:57-68.

Rafi SA, Nath UK. Variability, heritability, genetic advance and relationship of yield and yield contributing characters in dry bean (Phaseolus vulgaris L.). Journal of Biological Sciences. 2004; 4:157-159.

Rani KR, Raju CS, Reddy KR. Variability, correlation and path analysis studies in bitter gourd (Momordica charantia L.). Agricultural Science Digest; 2015; 35(2):106-110.

Rohman MM, Iqbal ASM, Arifin MS, Akhtar $Z$, Husanuzzaman M. Genetic variability, correlation and path analysis in mungbean. Asian Journal of Plant Sciences. 2003; 2(1724):1209-1211.

Singh B, Pandey VP, Kumar S. Genetic variability, correlation and path coefficient analysis in bitter gourd (Momordica charantia L.), New Agriculturist. 2012; 23(2): 239-244.

Tyagi, Nidhi., Singh, V.B., and Maurya, P.K. (2018). Character association and path coefficient analysis of bitter gourd (Momordica charantia L.) genotypes, Journal of Pharmacognosy and Phytochemistry, 7(2): 2419-2422

Yadagiri J, Gupta NK, Tembhre D, Verma S. Genetic variability, correlation studies and path coefficient analysis in bitter gourd (Momordica charantiaL.). Journal of Pharmacognosy and Phytochemistry. 2017; 6(2):63-66.

Zhang H, Schroder JL, Fuharman JK, Basta NT, Storm DE, Payton ME. Path and multiple regression analyses of phosphorus sorption capacity. Soil Science Society of America Journal. 2005; 69: 96-106.

\section{How to cite this article:}

Deepak Maurya, V.B. Singh, G.C. Yadav, VeerendraKumar, Shivam Dubey and Ashok Kumar Pandey. 2019. Study the Correlation Coefficient and Path Coefficient for the yield and yield Component of Bitter Gourd (Momordica charantia L.). Int.J.Curr.Microbiol.App.Sci. 8(02): 952-960. doi: https://doi.org/10.20546/ijcmas.2019.802.110 